\title{
UNITARITY BOUNDS FOR INELASTIC DIFFRACTION
}

\author{
L. CAṄESCHI, P. GRASSBERGER and H.I. MIETTINEN* \\ CERN, Geneva, Switzerland \\ and \\ F. HENYEY \\ Physics Department, University of Michigan, Ann Arbor, Michigan, USA
}

Received 4 March 1975

Revised manuscript received 5 April 1975

\begin{abstract}
Unitarity in the s-channel is invoked to derive an upper bound for the inelastic diffractive cross-section as a function of impact parameter. The application of this bound to high-energy proton-proton scattering strongly suggests that inelastic diffraction should be peripheral in the impact parameter space.
\end{abstract}

Introduction. Recent observation of a structure around $t=-0.2 \mathrm{GeV}^{2}$ in the differential cross-section of the process $p p \rightarrow p\left(n \pi^{+}\right)$at the CERN ISR [1] supports the assumption that inelastic diffraction, unlike elastic diffraction, is peripheral in the impact parameter space. This assumption has been made very plausible in the past by many authors [2-6], partially on intuitive grounds, partially on the basis of phenomenological analyses of experimental data.

In this letter we suggest that the previous conclusion is in fact a very natural consequence of $s$-channel unitarity and of the assumption that inelastic, as well as elastic diffraction, is the shadow of non-diffractive particle production. Using these assumptions, Pumplin has recently derived the following bound [7]:

$\sigma_{\mathrm{el}}(b)+\sigma_{\text {diff }}(b) \leqslant \frac{1}{2} \sigma_{\text {tot }}(b)$.

Here, $\sigma_{i}(b) \equiv(1 / \pi) \mathrm{d} \sigma_{i} / \mathrm{d} b^{2}$, where $i=$ el, diff, tot, is the elastic, inelastic diffractive and total cross-section, respectively, in a collision at the impact parameter $b$. We apply this bound to high-energy proton-proton scattering and find that it is indeed very restrictive. In particular, it implies that at $b=0$ the inelastic diffractive cross-section can be most a third of the elastic cross-section. Since the total inelastic diffractive crosssection is roughly equal to the elastic one (both are

* Herman Rosenberg Foundation Fellow. On leave of absence from the Research Institute for Theoretical Physics, University of Helsinki, Finland. around $7-8 \mathrm{mb}$ at the top ISR energy), the conclusion inevitably follows that inelastic diffraction must be more peripheral than elastic scattering.

We then examine in what circumstances the bound eq. (1) could be saturated. We derive the following stronger bound:

$$
\begin{aligned}
& \sigma_{\text {diff }}(b) \leqslant \frac{1}{4} \sigma_{\text {tot }}^{(1)}(b) \cdot \sigma_{\text {tot }}^{(2)}(b) \\
& \sigma_{\text {diff }}(b) \leqslant\left[1-\frac{1}{2} \sigma_{\text {tot }}^{(1)}(b)\right]\left[1-\frac{1}{2} \sigma_{\text {tot }}^{(2)}(b)\right]
\end{aligned}
$$

where both inequalities must be simultaneously satisfied. Here $\sigma_{\text {tot }}^{(1)}$ is the proton-proton total cross-section and $\sigma_{\text {tot }}^{(2)}$ is a suitably defined average cross-section of inelastic diffractive states (resonant or non-resonant) on other such states and on protons. From eq. (2) it follows that the bound eq. (1) can be saturated only if, for all $b, \sigma_{\text {tot }}^{(1)}(b)+\sigma_{\text {tot }}^{(2)}(b)=2$. Strictly speaking, this would imply that $\sigma_{\text {tot }}^{(2)}$ is infinite. In practice one can essentially saturate the integrand bound if this relation holds for a large range of $b$, say $b<2 \mathrm{fm}$, but the shape of $\sigma_{\text {tot }}^{(2)}(b)$ required to achieve this result is obviously very peculiar.

In order to find a more physical bound, we require that $\sigma_{\text {tot }}^{(2)}(b)$ is a smoothly decreasing function of $b$ (such as a Gaussian, say) and that the integrated cross-section $\sigma_{\text {tot }}^{(2)}$ should not be unreasonably large. It turns out that eq. (2), when supplemented by these assumptions, provides us with a very strong bound for $\sigma_{\text {diff }}$. This bound has a sharply peripheral shape in $b$ - 
space, and it restricts $\sigma_{\text {diff }}$ to be $\$ 8 \mathrm{mb}$. Since the experimental value of $\sigma_{\text {diff }}$ is around $8 \mathrm{mb}$, we conclude that the above bound should be nearly saturated at all $b$-values that contribute appreciably to the integrated cross-section and, consequently, that inelastic diffraction should be peripheral in $b$-space.

General bound. Let us recapitulate Pumplin's derivation of the bound eq. (1). We introduce the notation $S=1+2 \mathrm{i} T=1-2 t$.

Here, $S$ is the $S$-matrix. We consider the collision of two protons at high energies, and assume that there are an unspecified, but finite, number of diffractive states produced (this is only for convenience in presentation). We choose a basis where these states are the $N$ first ones, and write the $t$-matrix on this basis:

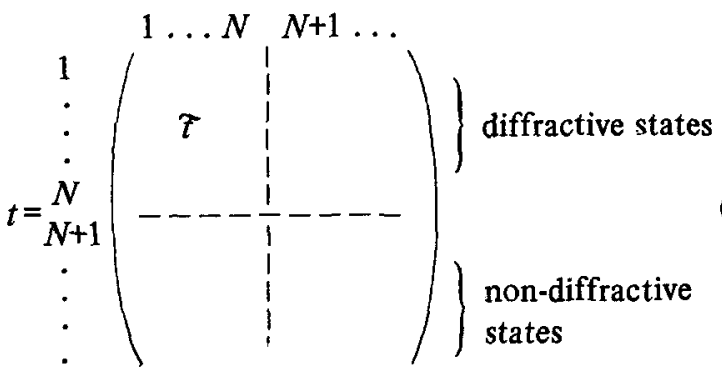

This representation defines a sub-matrix $\widetilde{\tau}$, whose elements describe the transitions among the diffractive states. We assume, for simplicity, the diffractive amplitudes $T_{i j}(i, j=1, \ldots, N)$ to be purely imaginary. Consequently, $\tilde{t}$ is a real symmetric matrix. Since the eigenstates of $\mathcal{Z}$ are linear combinations of the original diffractive states, they are diffractive, too. Thus, we may assume that elastic scattering of these eigenstates is purely absorptive. This implies that the eigenvalues of $\tilde{Z}$ should be real numbers between zero and one half:

$0 \leqslant \lambda_{n} \leqslant \frac{1}{2}, \quad n=1, \ldots, N$.

Our normalization is such that $\lambda_{n}=\frac{1}{2}$ corresponds to total absorption of the $n$th eigenstate.

Since $\tilde{T}$ is a real symmetric matrix, the transformation $U$ which diagonalizes it is unitary. Thus, we get

$(\mathcal{T})_{11}=\sum_{n=1}^{N}\left|U_{1 n}\right|^{2} \lambda_{n}$,
$\left(\check{t}^{2}\right)_{11}=\sum_{n=1}^{N}\left|U_{1 n}\right|^{2} \lambda_{n}^{2}$.

Here, $|1\rangle$ is the incoming two-particle state.

Combining eqs. (5) and (6), the following inequality is obtained:

$(\mathfrak{F})_{11} \geqslant 2\left(\mathfrak{T}^{2}\right)_{11}$.

The total, elastic and inelastic diffractive cross-sections are

$\sigma_{\text {tot }}=4 \widetilde{\tau}_{11}$.

$\sigma_{\mathrm{el}}=4\left(\tilde{t}_{11}\right)^{2}$

$\sigma_{\text {diff }}=4 \sum_{n=2}^{N}\left(\mathcal{f}_{1 n}\right)^{2}$.

Neglecting spin, it follows from angular momentum conservation that all the above relations are valid for each impact parameter separately. The bound eq.

(1) follows then directly from eqs. (7) and (8).

Application of eq. (1). Let us now apply eq. (1) to high-energy proton-proton scattering. We first integrate it, and obtain for the total inelastic diffractive cross-section the upper bound $\sigma_{\text {diff }} \leqslant \frac{1}{2} \sigma_{\text {tot }}-\sigma_{\text {el }}$. At the top ISR energy, $1500 \mathrm{GeV} / c$, the total and elastic cross-sections are approximately $43.5 \mathrm{mb}$ and $8.5 \mathrm{mb}$, respectively. Inserting these values into our inequality, we get a value for the upper bound of $\sigma_{\text {diff }}$ about $13 \mathrm{mb}$. At present, the most reliable estimates for $\sigma_{\text {diff }}$ are obtained from triple-Regge analyses of the inclusive spectra of $p p \rightarrow p+X$. Such analyses have been carried out by several authors [8]. Although the individual analyses differ in their assumptions and, in particular, in their results concerning the decomposition of the diffractive spectra into their scaling and nonscaling components, their estimates for the total amount of inelastic diffraction seem to agree reasonably well. The value of $\sigma_{\text {diff }}$ is $7-8 \mathrm{mb}$ at $1500 \mathrm{GeV} / c$. Hence, the integrated bound $13 \mathrm{mb}$ is far from being saturated.

Nevertheless, the differential bound eq. (1) seriously restricts the amount of inelastic diffraction at small values of $b$. In fact, inserting in eq. (1) the values of $\sigma_{\text {tot }}(b)$ and $\sigma_{\mathrm{el}}(b)$ taken from a recent analysis of ISR elastic-scattering data [9], we obtain for $\sigma_{\text {diff }}(b)$ the 


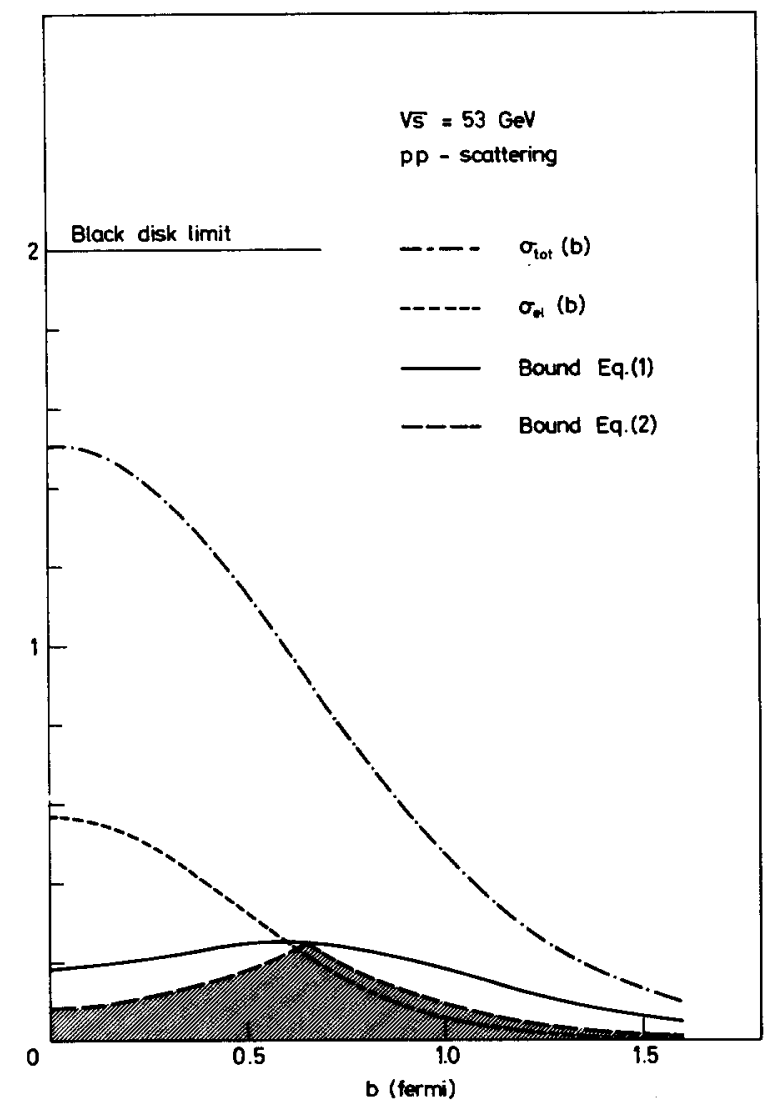

Fig. 1. Upper bounds for the cross-section of inelastic diffraction $\sigma_{\text {diff }}(b)$ versus $b$, for pp scattering at $\sqrt{s}=53 \mathrm{GeV}$. - : bound given by eq. (1). --- : bound calculated from eq. (2) with Gaussian $\sigma_{\text {tot }}^{(2)}(b)$ and $\sigma_{\text {tot }}^{(2)}=86 \mathrm{mb}$. Shown are also the impact parameter distributions for the pp total $(-\cdot-.-)$ and elastic (-- - -) cross-sections.

bound shown in fig. 1. Although we know that this bound is not saturated for all values of $b$, it is so restrictive at small $b$ that an integrated value of $\sigma_{\text {diff }} \sim 7 \mathrm{mb}$ can be obtained only if $\sigma_{\text {diff }}(b)$ is much more peripheral than $\sigma_{\mathrm{el}}(b)$.

Next we shall examine, under what conditions the bound eq. (1) could be saturated at all impact parameters. For this purpose, it is convenient to examme$\widetilde{t}$ in a basis in which state $|1\rangle$ is the elastic state; state 12〉, defined by

$|2\rangle=\frac{2}{\sqrt{\sigma_{\text {diff }}}}[\tilde{Z}|1\rangle-|1\rangle\langle 1|\widetilde{Z}| 1\rangle]$,

is the linear superposition of diffractive states ob- tained in pp scattering, and the remaining states $|3\rangle, \ldots,|n\rangle$ are chosen so as to complete the basis. According to our assumption on the shadow nature of diffraction scattering, also the eigenvalues of the $2 \times 2$ matrix

$$
2\left(\begin{array}{ll}
\tau_{11} & \tau_{12} \\
\tau_{21} & \tau_{22}
\end{array}\right)=\left(\begin{array}{cc}
\frac{1}{2} \sigma_{\text {tot }}^{(1)}(b) & \sqrt{\sigma_{\text {diff }}(b)} \\
\sqrt{\sigma_{\text {diff }}(b)} & \frac{1}{2} \sigma_{\text {tot }}^{(2)}(b)
\end{array}\right)
$$

must be real and in the range 0 to 1 . This requirement yields immediately eq. (2) $\neq$.

The bound eq. (1) follows from eq. (2) and can be saturated only if both inequalities eq. (2a) and eq. (2b) are simultaneously saturated. This happens when

$\sigma_{\text {tot }}^{(1)}(b)+\sigma_{\text {tot }}^{(2)}(b)=2$,

i.e., when the sum of the two total cross-sections saturates the black disk limit. Experimentally, $\sigma_{\text {tot }}^{(1)}(b)$ is very much like a Gaussian. Then, in order for eq. (9) to be valid, $\sigma_{\text {tot }}^{(2)}(b)$ must be an increasing function of $b$, and at large impact parameters it must approach, and finally saturate, the black disk limit. Such a behaviour of $\sigma_{\text {tot }}^{(2)}(b)$ that corresponds, in particular, to an infinite cross-section for the state 2 , is physically unacceptable. Consequently, we conclude that the bound eq. (1) cannot be saturated at all impact parameters.

More stringent bounds. To find a more physical bound, we impose that $\sigma_{\text {tot }}^{(2)}(b)$ [in analogy to $\sigma_{\text {tot }}^{(1)}(b)$ ] has a central shape (i.e., is a monotonically decreasing function of $b$ ). For simplicity, we parametrize it as a Gaussian ${ }^{\neq}$. We then analyse the bound on $\sigma_{\text {diff }}$ as a function of $\sigma_{\text {tot }}^{(2)}$.

* Stronger bounds could be obtained by imposing that all $N$ eigenvalues of the complete $\tilde{f}$ matrix are in the range $0-\frac{1}{2}$. In practice, it is hard to exploit these bounds, since they depend on unknown transition amplitudes between inelastic diffractive states.

\# In order to test the sensitivity of our results to the parametrization used for $\sigma_{\text {tot }}^{(2)}(b)$ we have repeated our calculations using several reasonable functions to describe $\sigma_{\text {tot }}^{(2)}(b)$. For all cases we tried, the results were very similar to those obtained using the Gaussian parametrization, both in their shape and in their magnitude. Hence, we may conclude that the bound is not sensitive to the exact shape of $\sigma_{\text {tot }}^{(2)}(b)$. We point out that our assumption that $\sigma^{(2)}(b)$ is central is not equivalent to assuming that all the total cross-sections of the "physical" diffractive states are central, since the state $|2\rangle$ is a superposition of the previous ones with $b$-dependent coefficients. 


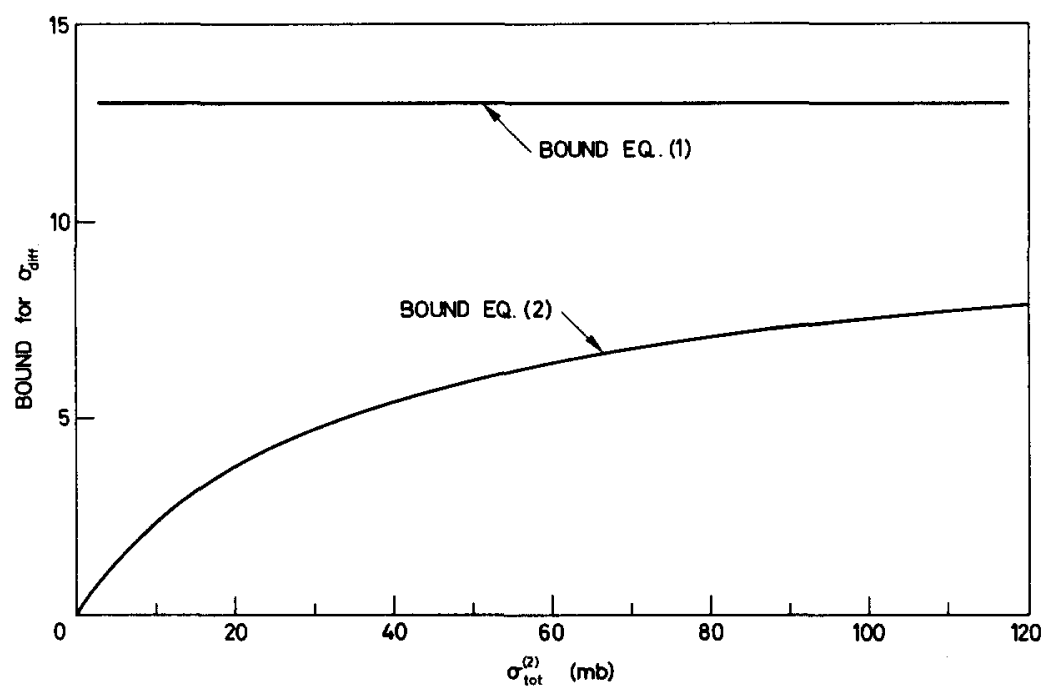

Fig. 2. Upper bound for the integrated cross-section of inelastic diffraction versus $\sigma_{\text {tot }}^{(2)}$, for pp scattering at $\sqrt{s}=53 \mathrm{GeV}$.

The result is shown in fig. 2 . From it we learn that a value $\sigma_{\text {diff }} \approx 8 \mathrm{mb}$ can be obtained only if $\sigma_{\text {tot }}^{(2)}$ is at least twice as large as the pp total cross-section. This result may be interpreted as providing some evidence that the average total cross-section of inelastic diffractive states on other such states and on nucleons is larger than the proton-proton total cross-section. Total cross-sections of low-mass diffractive states on nucleons have been measured in experiments on nuclei [10]. They seem to be more or less the same as the corresponding particle-nucleon cross-sections. The low-mass states studied, however, build up only a fraction of $\sigma_{\text {diff }}$. If the heavy mass diffractive states have large cross-sections on nucleons, the average cross-section $\sigma_{\text {tot }}^{(2)}$ could still be considerably larger than the corresponding particle-proton cross-section. Alternatively a large value of $\tilde{t}_{22}$ could be produced by the collective effect of off-diagonal transition elements among "physical" diffractive states and thus not correspond to any large "physical" total crosssection.

In any case, barring an unacceptably large value of $\sigma_{\text {tot }}^{(2)}$, we see that the bound that we obtain is very close to the experimental value of $\sigma_{\text {diff }} \geq 7 \mathrm{mb}$. Hence the value of $\sigma_{\mathrm{diff}}(b)$ cannot deviate very much from the corresponding bound for all values of $b$ that contribute substantially. The impact parameter dependence of the bound is shown in fig. 1 , for $\sigma_{\text {tot }}^{(2)}=2 \sigma_{\text {tot }}^{(1)}=$
$86 \mathrm{mb}$. The bound is seen to be sharply peripheral, peaking at $b=0.6 \mathrm{fm}$ (this is the impact parameter value at which $\sigma_{\text {tot }}^{(1)}=1$ ). From the above considerations we conclude that the actual shape of $\sigma_{\mathrm{diff}}(b)$ must be very similar. In momentum space, assuming the amplitude to be dominantly non-spin-flip, such a shape yields a dip around $t=-0.2 \mathrm{GeV}^{2}$.

Another interesting observation can be made in the large- $b$ region (not visible in the figure). There the bound restricts $\sigma_{\text {diff }}(b)$ to be much smaller than $\sigma_{\text {inel }}(b)$. This shows that particle production at large impact parameters must be dominated by non-diffractive processes.

Discussion. The essential physics underlying the bounds eqs. (1) and (2) is s-channel unitarity. The constraint that elastic scattering of the diffractive eigenstates of the $S$-matrix should obey unitarity reflects itself in the physical diffractive amplitudes, forcing inelastic diffraction to be peripheral, but leaving elastic diffraction central.

A useful tool to study unitarity effects is provided by the eikonal representation. Let us define a $2 \times 2$ eikonal matrix $\Omega$ such that

$$
\left(\begin{array}{ll}
\widetilde{\tau}_{11} & \widetilde{\tau}_{12} \\
\tau_{21} & \tau_{22}
\end{array}\right)=\frac{1}{2}\left(1-\mathrm{e}^{-\Omega}\right) \text {. }
$$

If eq. ( $2 b$ ) is saturated - which seems to be nearly the 


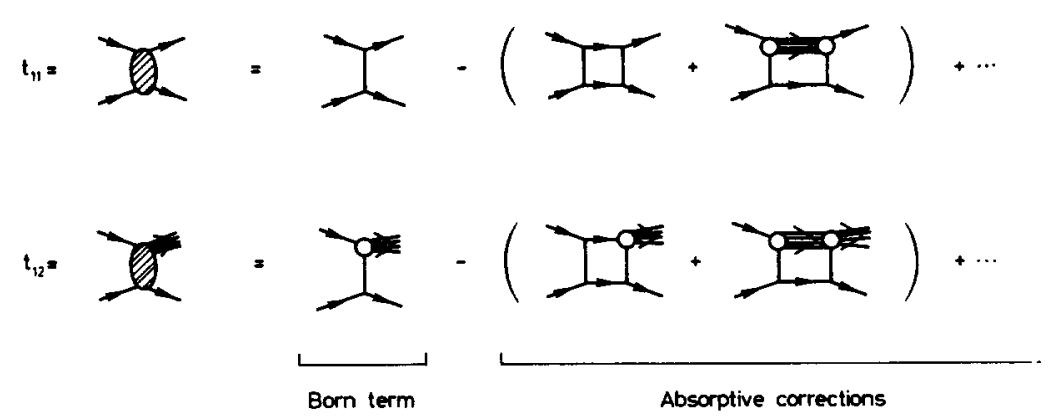

Fig. 3. Illustration of the unitarity structure of elastic and inelastic diffraction. The Born terms are built up by non-diffractive production.

case for $b \lessgtr 0.6 \mathrm{fm}-$ then $\mathrm{e}^{-\Omega}$ must have one eigenvalue equal to zero, corresponding to $\Omega_{i k} \rightarrow \infty$ in such a way that $\Omega_{12} \sim \sqrt{\Omega_{11} \Omega_{22}}$. Since, on the other hand, $\widetilde{t}_{12} \ll \sqrt{t_{11} \cdot \widetilde{t}_{22}}$, inelastic diffraction is much more strongly absorbed than elastic. This latter result is also found in the limit of weak absorption. If also in this case $\widetilde{t}_{12} \ll \widetilde{t}_{11} \sim \widetilde{t}_{22}$, we expand the amplitudes as (see fig. 3):

$$
\begin{aligned}
& 2 \tau_{11}(b)=\Omega_{11}(b)-\frac{1}{2}\left(\Omega_{11}^{2}(b)+\Omega_{12}^{2}(b)\right)+\ldots,(11 \mathrm{a}) \\
& 2 \tau_{12}(b)=\Omega_{12}(b)-\frac{1}{2} \Omega_{12}(b)\left(\Omega_{11}(b)+\Omega_{22}(b)\right)+\ldots .
\end{aligned}
$$

Since now $\Omega_{i k}(b) \approx \tau_{i k}(b)$, we expect $\Omega_{12}(b) \ll$ $\Omega_{11}(b) \approx \Omega_{22}(b)$. This means that the absorption (relative to the Born term) is about twice as strong for inelastic diffraction as for elastic scattering.

Finally, let us remark on diffraction dissociation in pion-nucleon and kaon-nucleon collisions. The unitarity mechanism discussed in this paper gives rise to strong absorption in inelastic diffraction only when $\sigma_{\text {tot }}(b)$ is much larger than one. The recent $\pi \mathrm{N}$ and KN elastic-scattering data from Fermilab [11] show that, in both cases, $\sigma_{\text {tot }}(b=0)$ is around one only. Hence our mechanism is not particularly effective in $\pi \mathrm{p}$ or $\mathrm{Kp}$ dissociation. Consequently, if our mechanism is the only source of peripherality in diffraction dissociation, the dip structure at small $t$-values should be absent or less pronounced in $\pi \mathrm{p}$ and $\mathrm{Kp}$ diffraction dissociation.
We are grateful to Dr. R. Phillips and Professors V. Ruuskanen and L. Van Hove for useful discussions. One of the authors, H.I.M., thanks the Herman Rosenberg Foundation for financial support.

\section{References}

[1] E. Nagy et al., CERN preprint, Experimental results on inelastic diffraction scattering in proton-proton collisions at the ISR (1974).

[2] G.L. Kane, Acta Phys. Polon. B3 (1972) 845.

[3] N. Sakai and J.N.J. White, Nucl. Phys. B59 (1973) 511.

[4] H.I. Miettinen, Contribution to the 9 th Rencontre de Moriond, 1974, preprint CERN-TH 1864 (1974).

[5] W. Cheng, J.K. Walker and T.T. Wu, Phys. Rev. D9 (1974) 749.

[6] S. Humble, Nucl. Phys. B76 (1974) 137; B86 (1975) 285.

[7] J. Pumplin, Phys. Rev. D8 (1973) 2899; R. Blankenbecler, J.R. Fulco and R.L. Sugar, Phys. Rev. D9 (1974) 736, and references given therein.

[8] R.D. Field and G.C. Fox, Nucl. Phys. B80 (1974) 367 ; D.P. Roy and R.G. Roberts, Nucl. Phys. B77 (1974) 240.

[9] P. Pirilä, P.V. Ruuskanen and H.I. Miettinen, to be published. Some preliminary results of this analysis are given in ref. [4].

[10] For a review see, for example, J.L. Rosen, Proc. XVII Intern. Conf. on High-energy physics, London, 1974 (Rutherford Lab., Chilton, Didcot, 1974), p. I.16.

[11] C.W. Akerlof et al., Michigan preprint UM-HE-74-20 (1974). 